Medicina et Pharmacologia Experimentalis 1965;13:I-VII

\title{
Contents, Vol. 13, 1965
}

International Journal of Experimental Medicine Internationale Zeitschrift für experimentelle Medizin Journal International de Médecine expérimentale Editor principalis R. DOMENJOZ, Bonn

Edítores

C. H. BEST, Toronto B. B. BRODIE, Bethesda, Md.

J. CHEYMOL, Paris L. HEILMEYER, Freiburg i. Br.

B. MUKERJI, Lucknow A. VANNOTTI, Lausanne

$1965 \quad|* *| \quad$ Vol. 13

BASEL (Schweiz) S. KARGER NEW YORK

COLLABORATORES

H. H. ANDERSON, San Francisco, Calif.; B. ANGELOPOULOS, Athen ; S. V. ANICHKOV, Leningrad; R. B. ARORA, New Delhi; G. ASTALDI, Tortona; C. B. BALLABIO, Milano ; T. BANDO, Tokyo; R. M. BERGSTRÖM, Helsinki; F. BERNHEIM, Durham, N.C.; J. R. BOISSIER, Paris; S.BONFILS, Paris; K.BUCHER, Basel; 0. BUCHER, Lausanne; J. BÜCHI, Zurich; J. J. BURNS, Tuckahoe, N.Y.; N.P.BUU-HOI, Paris; R.CAHEN, Maisons Alfort (Seine); J. CARLIER, Liège; F. CAUJOLLE, Toulouse; A. CERLETTI, Basel;

E.CHAPADEIRO.Uberada, Brazil; R.CHAUSSINAND, Paris; R.COIRAULT, Paris; S. COURVOISIER, Vitry; W. CUTTING, Honolulu, Hawai; W. DAMESHEK, Boston, Mass.; M. H. DRAPER, Edinburgh; T. DURAN QUEVEDO, Habana; H. DÜKER. Marburg/Lahn; W. EGER, Göttíngen; E. EICHENBERGER, Bern; P. F AVAR GER, Geneve; H. FERNER, Heidelberg; A. FLECKENSTEIN, Freiburg i. Br.; R.FONTAINE, Strasbourg; H. FRIEBEL, Heidelberg; E.FROMMEL, Geneve; S. GARATTINI, Milano; J. GARRETT, Porto; H. GARTNER, Kiel; F. GEORGI, Basel; Ch. GERNEZ-RIEUX, Lille; B. P. GHOSH, Calcutta; C. GIURGEA, Bruxelles; E. GRANDJEAN, Zurich; S. GRISOLIA, Kansas City, Kan.; E. GRÜNTHAL, Waldau-Bern; D. GUEVARA, Granada; G. L. HABERLAND, Elberfeld; B. N. HALPERN,Paris; F. HAUSCHILD, Leipzig;

F. HEIM, Erlangen; H.-J. HEITE, Freiburg i. Br.; J. HUFSCHMIDT, Würzburg; C. G. HUNTER, London;

B. ISSEKUTZ, Budapest; I. IVANCEVIC, Zagreb; J. A. IZQUIERDO, Buenos Aires ; J.JACOB, Paris;

P. JANSSEN, Beerse (Belgique) ; K. JUNKMANN, Berlin; K. KARZEL, Bonn; E. KELEMEN, Szeged;

H. KLIEWE, Mainz; W. KOLL, Göttingen ; T. KOPPANYI, Washington, D.C. ; M.KRAMER, Berlin; 
E. KREPPEL, Bonn; G. KUSCHINSKY, Mainz; H. LABORIT, Paris; H. LANGECKER, Berlin;

G. LATORRE, Medellin; W. L $\Lambda$ UBENDER, Frankfurt/Main ; P. LECHAT, Paris ; J.

LEVY, Paris;

J.LINDNER, Hamburg; A. LOUBATIÈRES, Montpellier; G. LUDANY, Budapest; H. MAIEER-

BODE, Bonn;

J.MARDONES, Santiago; L. C. MARK, New York, N.Y.; A. S. MARRAZZI, Pittsburgh, Pa.;

H. MATHIES,

München; P. DI MATTEI, Milano; J. L. MELNICK, Houston, Texas; K. MÖRSDORF, Bonn; S. NEUKOMM,

Lausanne; O. NIESCHULZ, Hamburg; W. OELSSNER, Dresden; G. ORZECHOWSKI, Kòln;

H. OSSWALD,

Heidelberg; W. OSSWALD, Porto; G.PANEQUE, Sevilla; K. PANAGOPOULOS, Athen; A. PLETSCHER,

Basel; A. R. RATSIMAMANGA, Paris; J. L. R-CANDELA, Madrid; F. REZZESI, Parma; M. ROCHA E SILVA,

São Paulo; R. RONDEZ, Zurich; J. R. RÜTTNER, Zurich; F. SCHEIFFARTH, Erlangen; G.

SCHÖN-

HOLZER, Bern; H. SELYE, Montreal; E. B. SIGG, Ardsley, N.Y.; J. STAHL, Strasbourg; H. STAUB, Basel;

J. STEPAN, Prag; P. STERN, Sarajevo; V. SUBBU, Guntur; W. TAUB, Rehovoth; W.

THEOBALD, Basel;

J.THUILLIER, Paris; F. TIEMANN, Bonn; J. TRIPOD, Basel; F. G.VALDECASAS, Barcelona; Y. VAL-

LAD ARES, Madrid; M. VERSTRAETE, Louvain ; T. WENSE, Innsbruck ; H. WESTLING, Göteborg;

W. WILBRANDT, Bern; G. WILHELMI, Basel; H. G.WILLIAMS-ASHMAN, Chicago, Ill.; W. WIRTH,

Elberfeld; E. A. ZELLER, Chicago, IU.

TRANSLATORES

English-French: Français-Allemand:

G. THUILLIER, Paris (France) W. THEOBALD, Basel (Schweiz)

English-German: Deutsch-Französisch:

K. MÖRSDORF, Bonn (Deutschland) P. LECHAT, Paris (France)

Deutsch-Englisch: J. WARD, Basel (Schweiz)

Alle Rechte, insbesondere das der Übcrsetzung in fremde Sprachen, vorbehalten.

Ohne ausdrückliche Genehmigung des Verlages ist es auch nicht gestattet, dieses Buch oder

Teile daraus auf pholomechanischem Wege (Photokopie, Mikrokopie) zu vervielfẫltigen.

(C)

Copyright 1965 by S. Karger AG., Basel Printed in Switzerland by Buchdruckerei Friedrich

Reinhardt AG., Basel Cliches: Aberegg-Steiner \& Cie. AG., Bern, und Steiner \& Cie. AG., Basel Index

Acevedo, Ximena: vide Muñoz, Carlos

Abou-Rebyeh, L.: vide Warnatz, $\mathrm{H}$.

Abreu, M. L.: vide Guedes, M. F.

Ammon, II. P. T.: vide Heim, F. 
Arora, H. R. K.: A Study on the Effects of Polyene Antifungal Antibiotics on the Per fused Guinea-pig Heart 57

Arora, H. R. K.: Effects of Polyene Antibiotics on the Heart; A Study with Nystatin and Lagosin 155

Auerswald, W. and Doleschel, W.:

A Shock-Producing Factor Contained in Human Placental Protein

Derivatives 303

Bartonícek, V.: Increase of Serotonin Levels in the Specific Serotonergic Neurons of Rat Brain Caused by Phenelzine 184

Benyó, I.; Füsy, Fr.; Görgõ, P.; Ludány, G. und Mitsányi, A.:

Die Wirkung der Duodenumsäuerung auf den Leberkreislauf ... 317

Berger, F. M.; Kletzkin, M. and Margolin, S.:

The Combined Action of Meprobamate and Benactyzine 103

Berti, F.; Lentati, R. and Usardi, M. M.:

Effects of Cold Exposure on Heart Function in Immunosympathec-

tomized Rats 227

Berti, F.; Lentati, R. and Usardi, M. M.:

The Species Specificity of Prostaglandin Ex Effects on Isolated Heart 233

Bonfils, S.; Gros, M.; Caulin, Ch.; Dubrasquet, M. et Potet, F.:

Techniques pour l'obtention de pancréatites aiguë et chronique chez

le rat 262

Bose, S. N.: Chemical Method for the Detection of Ovulation Time (III) .... 250

Breining, $\mathrm{H}$. und Strubelt, 0 :

Die Bedeutung der adrenergischen /3-Rezeptoren für die cardiotoxi-

sche Wirkung sympathicomimetischer Amine $\quad 169$

Burkhard, W. P.; Gey, K. F. and Pletscher, A.:

Inhibition of Tryptophan and Phenylalanine Hydroxyïase by $\alpha$ -

Methyldopa, $\alpha, /$, ,/?-Trimethyldopa, and Other Compounds 383

Buu-Hoï, N. P. et Mabille, Ph. :

Difference intéressante d'action biologique entre le 12-acétyl-6-amino-

chrysène et le 12-isovaléroyl-6-aminochrysène

Cannon, J. F.: The Treatment of Pituitary Response Diabetes Insipidus

Caulin, Ch. : vide Bonfils, S.

Cebecauer, L.: vide Trnavský, K.

Cheymol, G.: Sur Tactivité pharmacologique de derives phosphorés du penta-ery-

thritol 117

Clopotaru, M.: vide Saragea, M.

Combet Farnoux, Cl. :

vide Gautier, J. A.

Doleschel, W.: vide Auerswald, W.

Dubrasquet, M.: vide Bonfils, S.

Eckmann, F. und Immich, H.:

Neuroleptica und Sauerstoffmangelbeatmung

Eger, W. und Fernholz, J.:

Über den thyreostatischen Effekt des Phenylbutazon und des Oxy-

phenbutazon unter dem Einfluß des Trijodthyronin 
Estler, C. J.: vide Heim, F.

Favarger, P.: vide Geissbühler, P.

Favarger, P.: vide Rous, S.

Fernholz, J.: vide Eger, W.

Finger, K. und Zicha, L.:

Dünnschichtchromatographische Untersuchungen über Oxyphenbu-

tazon in Körperflüssigkeiten 161

Fontaine, L.; Grand, M.; Quentin, Y. et Merle, S.:

Activité anti-inflammatoire comparée de substances anticoagulantes;

Mode d'action de la Phénylindanedione 137

Formanek, K.; Holler, H. J. A. und Peterlik, M.:

Zur Isolierung und Charakterisierung eines neuen Serumfaktors, der

rhythmische Uteruskontraktionen auslöst. I. Mitteilung 331

Formanek, K.: Die Wirkung von Saluretika auf experimentell erzeugte Odeme der

Rattenpfote 353

Fregnan, G. B. and Glässer, A. H.:

Synergistic Action of an Anti-5-Hydroxytryptamine, Anti-Hista-

mines and Cortisone in Experimental Anaphylaxis 189

Füsy, Fr.: vide Benyó, I.

Gautier, J. A.; Combet Farnoux, Cl.; Nakajima, H. et Thuillier, J.:

Activité anesthésique locale d'une série d'amino-alcools et amino-cé-

tones aromatiques à chaîne butylique. Etude des relations entre la

structure et Гactivité de ces composes $\quad 325$

Geissbühler, P. et Favarger, P.:

Effet du ribose sur Tincorporation du bicarbonate $\mathrm{C} 14$ dans les acides

gras 7

Genetet, B.: vide Larcan, A.

Gey, K. F.: vide Burkhard, W. P.

Giannitsis, D. I.: Biochemische Untersuchungen des Histons der Leukozytenkerne beim Schwein 206

Glässer, A. H.: vide Fregnan, G. B.

Goñi, P. M.: vide Quijada, C. L.

Görgó, P.: vide Benyó, I.

Grand, M.: vide Fontaine, L.

Gros, M.: $\quad$ vide Bonfils, S.

Guedes, M. F.; Santos Mota, J. M. and Abreu, M. L.:

Some Technical Problems of the Thioctic Acid Microbiological Assay

in Human Serum

1

Haefely, W.: vide Thoenen, $\mathrm{H}$.

Heim, F.; Ammon, H. P. T.; Estler, C. J. und Mikschiczek, D.:

Funktion und Stoffwechsel des Gehirns unter Einwirkung niedriger

Alkoholkonzentrationen $\quad 361$

Holler, H. J. A.: vide Formanek, K.

Homolka, J.: vide S $\tau$ ÈpÁN, J.

Hürlimann, A.: vide Thoenen, H.

Immich, H.: vide Eckmann, F. 
Izquierdo, I.: vide Merlo, A. B.

Kakác, B.: $\quad$ vide Stëpán, J.

Karrer, K. and Rubini, J. R.:

On the Fate of Labelled Ehrlich Ascites Cells in Mice, an Autoradio-

graphic Study Using H3 Thymidine 124

Karzel, K.: vide Witthaus, G.

Kletzkin, M.: vide Berger, F. M.

Larcan, A.; Streiff, F.; Peters, A. et Genetet, B.:

Action de $\Gamma$ Héparíne sur la viscosité sanguine

Lechaï, P.; Streichenberger, G. et Maget, E.:

Etude pharmacologique du réendormissernent provoqué chez le rat

par variation brusque de temperature ambiante 271

Lentati, R.: vide Berti, F.

Ludány, G.: vide Benyó, I.

LiJTHi, L.: vide Rous, S.

Mabille, Ph. : vide Buu-Hoï, N. P.

Maes, $H .:$ vide $\Lambda$ "erstraete, $M$.

Maget, E.: $\quad$ vide Lechat, $P$.

Margolin, S.: vide Berger, F. M.

Mathur, Raghubansh Bahadur: vide Selye, $\mathrm{H}$.

Merlo, A. B. and Izquierdo, I.:

Effects of Inhibitors of O-Methyl-Transferase and of Adrenergic

Blocking Agents on Conditioning and Extinction in Rats 217

Merle, S.: vide Fontaine, L.

Mikschiczek, D.: vide Heim, F.

Mitsányi, A.: vide Benyó, I.

Muñoz, Carlos; Paeile, Carlos, and Acevedo, Ximena:

Quantitative Studies on the Influence of Haloperidol on the Analgesic

and Electroencephalographic Effects of Morphine in Rabbits. ... 371

Nakajim4, H.: vide Gautier, J. A.

Negru, T.: $\quad$ vide Saragea, M.

Nicák, A.: The Influence of Serotonine and Amphetamine on Analgesic Effect of

Morphine after Reserpine Premedication in Rats and Mice ...

43

Nieschulz, 0.: Über die Merkfähigkeit bei Mäusen und ihre experimentelle Beein-

flußbarkeit 294

Paeile, Carlos: vide Muñoz, Carlos

Paluska, E.: vide Stëpán, J.

Pankow, D. und Peters, J. E.:

Über die hypokaliämische Wirkung von Natrium-p-aminosalicylat . 312

Peterlik, M.: vide Formanek, K.

Peters, J. E.: vide Pankow, D.

Peters, A.: $\quad$ vide Larcan, A.

Pletscher, A.: vide Burkhard, W. P.

Potet, $\Gamma$.: $\quad$ vide Bonfils, S.

Quentin, Y.: vide Fontaine, L.

Quijada, C. L. and Goñi, P. M.: 
The Effect of Yeast Glutamate Dehydrogenase on Blood Ammonia Level Following C1NH4 Gavage in ECK Fistula Dogs

Reber, K. und Studer, A.:

Autohistoradiographischer Nachweis von Phenacetin oder Phenacetin-Abbauprodukten im Leber- und Nierenpigment Phenacetin-belasteter Katzen 257

Rotaru, N.: $\quad$ vide Saragea, M.

Rous, S.; Lüthi, L. et Favarger, P.:

Action du bicarbonate sur la synthèse des acides gras chez Гanimal à jeun 199

Rubini, J. R.: vide Karrer, K.

Santos Mota, J. M.:

vide Guedes, M. F.

Saragea, M.; Clopotaru, M.; SicÄ, M.; Vladutiu, A.; Negru, T. and Rotaru, N.:

Biochemical Changes Occurring in Animals with Experimental Al

lergic Encephalomyelitis 74

Scheiffarth, F.: vide Warnatz, $H$.

Selye, H.; Tuchweber, B. and Mathur, Raghubansh Bahadur:

Influence of Norepinephrine upon the Thrombohemorrhagic Action of

Various Agar Preparations

49

SicÄ, M.: $\quad$ vide Saragea, M.

Stèpán, J.; Homolka, J. and Paluska, E.:

Effect of Ultrasound on Sera of Patients with Articular Disease and

Its Investigation by Means of Immunoelectrophoresis and Electro-

phoretic-Polarographic Analysis

24

7/8TÈPÁN, J. and KakáC, B.:

Investigation of the Values for Plasmamucoid-Tyrosine Isolated from

Native and Sonicated Plasma in Patients with Articular Disease . . 33

Stheichenberger, G.:

vide Lechat, $\mathrm{P}$.

Streiff, F.: $\quad$ vide Larcan, A.

Strubelt, 0.: vide Breining, $H$.

Studer, A.: $\quad$ vide Reber, K.

Thoenen, H.; Hürlimann, A. and Haefely, W.:

The Effect of Angiotensin on the Response to Postganglionic Sympa

thetic Stimulation of the Cat's Spleen; Lack of Facilitation of Nor

epinephrine Liberation $\quad 379$

Thuillier, J.: vide Gautier, J. A.

Trnavský, K.; Trnavská, Z. and Cebecauer, L.:

The Influence of Sodium Salicylate on the Increased Solubility of Col

lagen in Lathyrism 98

Trnavská, Z.: vide Trnavský, K.

Tuchweber, B.: vide Selye, $H$.

Usardi, M. M.: vide Berti, F.

Vermylen, J.: vide Verstraete, M.

Vekstraete, M.; Maes, H. and Vermylen, J. : 
Aluminium Nicotinate as a Hypocholesterolemic Agent

Vlãdut $\pi$ j, A.: vide Saragea, M.

Warnatz, H.; Scheiffarth, F. und Abou-Rebyeh, L.:

Serologísclie Untersuchungen zum Nachweis eines zellulär fixierten

Antikörpers bei der Transplantationsimmunität $\quad 177$

Witthaus, G. und Karzel, K.:

Nucleotidverteilungsmuster eines Stammes in vitro gezüchteter Ehr-

lich-Ascitestumorzellen

65

Witt, G.: Die Veränderungen im Serumeiweiß-Spektrum des Menschen in Abhängigkeit vom Alter 241

Zicha, L.: vide Finger, $\mathrm{K}$.

BUCHBESPRECHUNGEN - BOOK REVIEWS - LIVRES NOUVEAUX

$63,131,215,287,347,409$

Index rerum. . Index nominum

414

422 